\title{
Comparison of Complications that Arise after DT and FCLT in the Treatment of Post-Acne Scars
}

\author{
Nikhita', Kallappa ${ }^{2}$, Karana Malhotra', Francis Abel Kunnath ${ }^{1}$ \\ ${ }^{1} J u n i o r$ Resident, Department of Dermatology, Venereology \& Leprosy, Navodaya Medical College, Raichur, Karnataka, ${ }^{2}$ Professor \& Head, Department of \\ Dermatology, Venereology \& Leprosy, Navodaya Medical College, Raichur, Karnataka.
}

\section{Abstract}

Background: Acne vulgaris is a common chronic skin disease involving blockage and/or inflammation of pilosebaceous units (hair follicles and their accompanying sebaceous gland). Acne can present - non-inflammatory lesions (open and closed comedones), inflammatory lesions (papules, pustules, and nodules), and varying degrees of scarring or a mixture of both, affecting mostly the face but also the back and chest. Acne leads to significant morbidity that is associated with residual scarring and psychological disturbances such as poor self-image, depression, and anxiety, which leads to a negative impact on quality of life. Subjects and Methods: Total 200 patients divided into two groups of 100 each. One group was treated with dermaroller every four weeks for a period of 24 weeks and the other group was treated with fractional $\mathrm{CO} 2$ laser again every four weeks for a period of 24 weeks. Results: Six patients in dermaroller and nine patients in fractional $\mathrm{CO} 2$ laser group developed hyperpigmentation post procedure. Conclusion: Itching is not seen as a complication in the treatment with derma-roller, but in case of fractional $\mathrm{CO} 2$ laser group, a very small percentage of patients experienced itching a few days after the treatment due to peeling off of the scab that was formed after treatment.

Keywords: Complications, DT and FCLT, Post-Acne Scars.

Corresponding Author: Dr. Nikhita, Junior Resident, Department of Dermatology, Venereology \& Leprosy, Navodaya Medical College, Raichur, Karnataka

Received: May 2019

Accepted: May 2019

\section{Introduction}

Acne vulgaris is an inflammatory process localized to the pilosebaceous units of the face, chest, upper arms, and back. Acne vulgaris is seen to be most amongst the age groups of 14-21, both in boys and girls. It has been reported to affect as many as $80 \%$ of adolescents and young adults. The incidence of acne scarring in the general population is between 1 and 11 percent, making the treatment of acne scarring a very relevant topic in dermatology today. the early preclinical inflammation in acne persists throughout the acne lesion's life cycle, from micro-comedones to closed comedones to inflammatory lesions and eventually to postinflammatory erythema (PIE), post-inflammatory hyperpigmentation (PIH) and scarring. ${ }^{[1]}$

During the evaluation, scars are visually inspected, palpated, and stretched. The strengths of this system include simplicity and ease of application in practice. However, in the observation of severe cases, different patterns are simultaneously present and may be difficult to differentiate and also it is not a very effective system in comparing two treatment modalities for which more quantification is required. ${ }^{[2]}$

Another classification system for acne scarring is the ECCA (Echelle d'Evaluation clinique des Cicatrices d'acné) for facial acne scarring which is a quantitative scale and is based on the sum of individual types of scars and their numerical extent. Scar types considered to be more visibly disfiguring were weighted more heavily. ${ }^{[3]}$

A semi-quantitative assessment of the number of each of these scar types was then determined with a four-point scale, in which 0 indicates no scars, 1 indicates less than five scars, 2 indicates between five and 20 scars, and 3 indicates more than 20 scars. With this method, the relative extent of scarring for each scar type was calculated. The total score can vary from 0 to 540.The potential advantages of this system include independent accounting of specific scar types, thereby providing for separate atrophic and hypertrophic sub scores in addition to total scores. Potential short-comings include restriction to facial involvement, time intensity and undetermined clinical relevance of score ranges. ${ }^{[4]}$

Another classification system proposed by Goodman and baron is the quantitative global acne scarring grading system, which is based on the type of scars and number of scars. This system assigns fewer points to macular and mild atrophic scores than moderate and severe atrophic scores.

The multiplication factor for these lesion types is based on the numerical range whereby, for one to ten scars, the multiplier is 1 ; for $11-20$ it is 2 ; for more than 20 it is 3 . This system is accurate and reproducible. It not only allows 
a better quantitative assessment of the scars before and after treatment but is also an ideal tool in comparing two treatment modalities. Therefore we adopted this classification system in our study to assess and evaluate the efficacy of resurfacing with fractional CO2 laser versus resurfacing with dermaroller in the treatment of post acne scars.

Microneedling is a relatively new minimally invasive procedure involving superficial and controlled puncturing of the skin by rolling with miniature fine needles. Over a short period of time, it has gained mass popularity and acceptance as it is a simple, cheap, safe, and effective technique requiring minimal training. ${ }^{[5]}$

The advent of the concept of microneedling dates back to 1995 when Orentreich and Orentreich described dermal needling in the form of subcision for scar treatment and then independently in 1997 by a plastic surgeon Camirand who used tattoo guns without ink to take-off tension from postsurgical scars. Microneedling technique was given further shape by a German inventor Liebl in 2000 and a plastic surgeon Fernandes in 2006 who self-designed a drum-shaped device with multiple fine protruding needles and used it for percutaneous collagen induction at the XII Congress of the International Society of Aesthetic Plastic Surgery in Paris,France. ${ }^{[6]}$

Lasers were introduced into the plastic surgery and dermatology world initially for the treatment of vascular lesions. The introduction of the carbon dioxide laser for skin resurfacing in the mid 1990s rapidly became popular and replaced chemical peels and dermabrasion in many practices. The carbon dioxide laser has a wavelength of $10,600 \mathrm{~nm}$, an absorbing chromophore of water, and is used to vaporize tissue. created a tissue exposure time of less than one millisecond, which allowed tissue ablation with limited residual thermal damage of approximately 75-100 $\mu \mathrm{m}$. Erbium-YAG lasers $(2940 \mathrm{~nm})$ were introduced around 2000 and marketed for superficial resurfacing. Erbium lasers have a higher water absorption coefficient than carbon dioxide lasers (about 10 times more efficient) and ablate tissue with much less thermal damage $(5-10 \mu \mathrm{m})$.

\section{Subjects and Methods}

This study was performed in accordance with standard clinical guidelines and in compliance with local regulatory requirements. This was a prospective, single center, randomized, comparative study of efficacy of resurfacing with fractional $\mathrm{CO} 2$ laser versus dermaroller therapy in the treatment of post acne scars. An ethical clearance was obtained before starting the study.

\section{Method of collection of data:}

The study included 200 patients with post acne scars attending the Outpatient Department of Dermatology, Venereology and Leprosy, Medical College Hospital and Research Centre. A total of 200 patients were enrolled in the study. They were divided into two groups of 100 each. One group was treated with dermaroller every four weeks for a period of 24 weeks and the other group was treated with fractional $\mathrm{CO} 2$ laser again every four weeks for a period of 24 weeks. All 200 patients did not complete the treatment .

\section{Inclusion criteria}

- Patients with acne scars under the groupings of a score > 3 points on Global Acne Scarring Classification Scale.

- Patients who were willing to undergo the procedure.

- Patients above 18 years

\section{Exclusion criteria}

- Patients below 18 years.

- Patients with acne scars under the groupings of a score < 3 points on Global Acne Scarring Classification Scale.

- Patients with active acne.

- Patients with active bacterial, viral, or fungal infections in the area to be treated.

- Patients who has taken isotretinoin within previous 6 months.

- Predisposition to keloid formation / hypertrophic scarring.

- Ongoing ultraviolet exposure or prior radiation therapy to treatment area.

- Pregnant and lactating mothers.

\section{Results}

In case of dermaroller treatment, it was seen that the majority of the patients were in the age group of 21 to 25 years and 26 to 30 years comprising $32.6 \%$ and $34.8 \%$ of each group respectively. Thus, on an aggregate together they made up $67.4 \%$ of the total patients in the dermaroller group. On the contrary, the least number of patients were in the $>35$ years age group comprising $2.2 \%$.

Similarly, age analysis of patients who underwent the fractional $\mathrm{CO} 2$ laser resurfacing treatment showed that the majority of the patients belonged to the age group of 26-30 years $(39.8 \%)$, followed by patients under the age group of 21-25 years $(35.5 \%)$. Together it comprised $75.3 \%$. This group a had the least number of patients in $<20$ years age group forming $1.1 \%$.

Thus, the age wise analysis for both groups showed a distribution pattern which was almost similar.

Table 1: Demographic details of the patient

\begin{tabular}{|l|l|l|l|l|}
\hline Age & & DT & & FCLT \\
\hline & Frequency & \% & frequency & \% \\
\hline$<=20$ & 5 & 5.6 & 1 & 1.1 \\
\hline $21-25$ & 29 & 32.6 & 33 & 35.5 \\
\hline $26-30$ & 31 & 34.8 & 37 & 39.8 \\
\hline $31-35$ & 22 & 24.7 & 19 & 20.4 \\
\hline$>35$ & 2 & 2.2 & 3 & 3.2 \\
\hline Total & 89 & 100 & 93 & 100 \\
\hline
\end{tabular}

The two groups who underwent the dermaroller and fractional $\mathrm{CO} 2$ laser resurfacing treatment were analyzed 
for male-female ratio. It was seen that $45 \%$ of the patients were male and $55 \%$ were females for the dermaroller treatment and in the case of fractional $\mathrm{CO} 2$ laser resurfacing treatment the number of females who underwent the treatment was $61.3 \%$ as compared to males who were $38.7 \%$.

Table 2: Comparison of gender distribution between DT and FCLT

\begin{tabular}{|l|l|l|l|l|}
\hline \multirow{2}{*}{ SEX } & \multicolumn{2}{|l|}{ DT } & \multicolumn{2}{l|}{ FCLT } \\
\cline { 2 - 5 } & Frequency & \% & Frequency & \% \\
\hline Male & 40 & 45 & 36 & 38.7 \\
\hline Female & 49 & 55 & 57 & 61.3 \\
\hline Total & 89 & 100 & 93 & 100 \\
\hline
\end{tabular}

The complications occurring due to dermaroller and the fractional $\mathrm{CO} 2$ laser treatment were statistically analyzed. The complications which were analyzed were occurrence of erythema (redness), pain, and hyperpigmentation.

The erythema in the post procedure period was seen in $93.3 \%$ of patients in dermaroller group and in $77.4 \%$ of patients in fractional $\mathrm{CO} 2$ laser group.

Patients in the dermaroller group comparatively experienced more pain during procedure and immediately post procedure than those in the laser group i.e. $88 \%$ of the patients experienced pain in dermaroller group and $63.4 \%$ patients experienced pain in the laser group.

Table 3: Pain after DT and FCLT
\begin{tabular}{|c|c|c|c|c|}
\hline & DT & HCLT \\
\hline Pain & Frequency & $\%$ & frequency & $\%$ \\
\hline YES & 78 & 88 & 59 & 63.4 \\
\hline NO & 11 & 12 & 34 & 36.6 \\
\hline Total & 89 & 100 & 93 & 100 \\
\hline P $=0.000<0.05$ Significant
\end{tabular}

Six patients in dermaroller and nine patients in fractional $\mathrm{CO} 2$ laser group developed hyperpigmentation post procedure.

Itching is not seen as a complication in the treatment with derma-roller, but in case of fractional $\mathrm{CO} 2$ laser group, a very small percentage of patients experienced itching a few days after the treatment due to peeling off of the scab that was formed after treatment.

\section{Discussion}

The age of the patients was recorded by using the data from the questionnaire. Age wise analysis for both groups showed a distribution pattern which was almost similar. It was seen that the majority of the patients were in the age group of 21 to 25 years and 26 to 30 years comprising $67.4 \%$ of the total patients in dermaroller group. Similarly in fractional $\mathrm{CO} 2$ laser group majority of the patients belonged to the age group of 26-30 years (39.8\%), followed by patients under the age group of 21-25 years (35.5\%).

It has been noted that acne is associated with specific age groups of patients in higher incidence whereas it is low in other age groups. Acne and its complications are more prevalent among teenagers and the young adults. Acne vulgaris affects majority of the teenage groups and the most sufferers belong to the age group of 12-24 years. ${ }^{[7]}$ The occurrence of acne reduces after age the of 25 years and the percentage of patients is as low as $8 \%$. Similarly in this study majority of the patients are in the age group of 20-30 years. The awareness of new treatment modalities like microneedling and laser too is more among these age groups, thus it is relevant that the percentage of patients under the age groups of 21-25 and 26 -30 years are higher.

The two groups who underwent the dermaroller and fractional $\mathrm{CO} 2$ laser resurfacing treatment were analyzed for male-female ratio. It was seen that $45 \%$ of the patients were male and $55 \%$ were females for the Derma-roller treatment and in the case of fractional $\mathrm{CO} 2$ laser resurfacing treatment the number of females who underwent the treatment was $38.7 \%$ compared to the males who were $61.3 \%$. The results indicated a higher prevalence of females undergoing treatment for post acne scars than the males.

The complications which were analyzed in this study were occurrence of erythema (redness), pain, and hyperpigmentation. It was seen that occurrence of hyperpigmentation post procedure were seen more in case of fractional $\mathrm{CO} 2$ laser than dermaroller therapy and the incidence of pain and discomfort during the procedure was experienced more with dermaroller than fractional $\mathrm{CO} 2$ laser. ${ }^{[8]}$

Erythema which was seen more in most of the dermaroller patients $(93 \%)$ and it was also seen in the laser group comprising $77.4 \%$ which was temporary and lasted for 2-3 days post procedure. Hyperpigmentation which was seen in around $9.7 \%$ of fractional $\mathrm{CO} 2$ laser patients and 5\% patients of dermaroller which resolved on its own within a period of 4-6 weeks without any sequelae. The complaint of pain was seen more in the dermaroller group of $88 \%$ and comprised of about $63 \%$ of patients in the dermaroller group which appeared during the procedure and lasted to a few hours after it.

Various studies on the efficacy of dermaroller and fractional $\mathrm{CO} 2$ laser in treatment of scars have been conducted in the past but very limited data is available on comparison between these two modalities. Both dermaroller and fractional $\mathrm{CO} 2$ laser have proven their efficacy in treatment of scars.

A comparative study conducted in 2017 between the two modalities of treatment showed that all patients in Dermaroller group improved of which $88 \%$ improved by at least one grade and $12 \%$ patients showed improvement of the scars by 2 grades in Goodman and Baron qualitative scar grading scale. All the patients in fractional CO2 laser group showed improvement, of which $80 \%$ improved by 1 grade and rest $20 \%$ by another 2 grades in Goodman and Baron qualitative scar grading scale.

A study on microneedling in 39 Patients with darker skin colors (types 3, 4 and 5) with acne scars in 2018, showed that it is an effective treatment for both acne scars and associated pigmentation in patients with dark skin color. 
The treatment appears to be safe apart from transient redness, mild dryness, and small hematomas. ${ }^{[9]}$ Transient erythema is the most common adverse event noticed. ${ }^{[10,11]}$ One such study conducted by Majid (2009) with 37 patients and it was seen that the dermaroller therapy was extremely effective in the removal of even severe scars. It has been described by Majid that "Dermaroller treatment thus has definite advantages such as its cheaper cost, the comparative ease of the overall procedure and also the minimal downtime associated with it". ${ }^{[12]}$

\section{Conclusion}

It was seen that complications such as of dotted pigmentation of laser and hyperpigmentation post procedure were seen more in case of fractional $\mathrm{CO} 2$ laser and the occurence of pain and discomfort during the procedure was experienced more with dermaroller therapy.

\section{References}

1. Connolly D, Vu HL, Mariwalla K, Saedi N. Acne ScarringPathogenesis, Evaluation, and Treatment Options. J Clin Aesthet Dermatol. 2017;10:12-23.

2. Kurokawa I, Danby FW, Ju Q, Wang X, Xiang LF, Xia L et al. New developments in our understanding of acne pathogenesis and treatment. Exp Dermatol. 2009 ;18:821-32

3. Alestas T, Ganceviciene R, Fimmel S, Müller-Decker K, Zouboulis
CC. Enzymes involved in the biosynthesis of leukotriene B4 and prostaglandin E2 are active in sebaceous glands. J Mol Med. 2006;84:75-87.

4. Zouboulis C C. Acne and sebaceous gland function . Clin Dermatol. 2004;22:360-6.

5. Nagy IA, Pivarcsi K. Propionibacterium acnes and lipopolysaccharide induce the expression of antimicrobial peptides and proinflammatory cytokines/chemokines in human sebocytes. Microbes and Infection. 2006; 8: 2195-205.

6. Kim J, Ochoa M T, Krutzik S R, Takeuchi O, Uematsu S, Legaspi, A $\mathrm{J}$ et al. Activation of toll-like receptor 2 in acne triggers inflammatory cytokine responses. J Immunol. 2002;169:1535-41.

7. Wolfram D, Tzankov A, Pülzl P, Piza-Katzer H. Hypertrophic scars and keloids - a review of their pathophysiology, risk factors, and therapeutic management. Dermatol Surg. 2009; 35:171-81.

8. Fabbrocini G, Vita V D , Cozzolino A, Marasca C, Mazzella C, Monfrecola A. The Management of Atrophic Acne Scars : Overview and New Tools. J Clin Exp Dermatol Res. 2012; S5:01-07.

9. Marshall CD, Hu MS, Leavitt T, Barnes LA, Lorenz HP, Longaker MT. Cutaneous Scarring: Basic Science, Current Treatments, and Future Directions. Adv Wound Care (New Rochelle). 2018;7:29-45.

10. Nilforoushzadeh MA, Faghihi G, Jaffary F, Haftbaradaran E, Hoseini SM, Mazaheri N. Fractional Carbon Dioxide Laser and its Combination with Subcision in Improving Atrophic Acne Scars. Adv Biomed Res. 2017;6:20.

11. Hantash BM, Bedi VP, Chan KF, Zachary CB. Ex vivo histological characterization of a novel ablative fractional resurfacing device. Lasers Surg Med.2007;39:87-95

12. Hantash BM, Bedi VP, Kapadia B, et al. In vivo histological evaluation of a novel ablative fractional resurfacing device. Lasers Surg Med.2007;39:96-107.

Copyright: () the author(s), 2019. It is an open-access article distributed under the terms of the Creative Commons Attribution License (CC BY 4.0), which permits authors to retain ownership of the copyright for their content, and allow anyone to download, reuse, reprint, modify, distribute and/or copy the content as long as the original authors and source are cited.

How to cite this article: Nikhita, Kallappa H, Malhotra K, Kunnath FA. Comparison of Complications that Arise after DT and FCLT in the Treatment of Post-Acne Scars. Asian J. Med. Res. 2019;8(2):DT01-DT04.

DOI: dx.doi.org/10.21276/ajmr.2019.8.2.DT1

Source of Support: Nil, Conflict of Interest: None declared. 